\title{
Blood testing for sensitivity, allergy or intolerance to food
}

\section{Elana Lavine MD}

Competing interests: Elana Lavine has received payment from King Pharma for a lecture on anaphylaxis and a workshop on epinephrine autoinjectors.

This article has been peer reviewed.

Correspondence to:

Dr. Elana Lavine,

e.lavine@utoronto.ca

CMAJ 2012. DOI:10.1503 /cmaj.110026

\section{Case 1}

A 35-year-old man has psoriatic arthritis. After starting treatment with methotrexate, he seeks the opinion of a holistic care provider, who suggests testing for "food sensitivity" to identify any foods that may be contributing to his symptoms. The patient is provided with a test report, which suggests that cows' milk and dairy products, wheat, eggs and many other foods should be avoided. The patient is told that food intolerances may be heritable and that he should consider testing his healthy young daughter.

\section{Case 2}

A four-year-old girl has flexural patches of pruritic skin since infancy, diagnosed as atopic dermatitis. Her mother is concerned about the safety of topical corticosteroids and limits their use. She visits a holistic care provider and is offered a blood test, described as "food allergy" testing, to identify any foods contributing to the child's eczema. After blood is drawn and analyzed, the test results highlight many foods the child should be avoiding, including eggs. The parents keep a vegetarian household, and the child does not eat any meat or fish. Eggs are a major component of the child's diet.

$\mathrm{H}$ ealth care providers, both traditional and complementary, may offer blood testing to identify foods playing a role in patients' illnesses. These two fictional cases highlight common scenarios in which patients may seek or be offered blood testing for possible reactions related to food intake. However, the distinc-

\section{KEY POINTS}

- Food sensitivity is a nonspecific term and not a synonym for food allergy.

- Food-specific immunoglobulin G (IgG) testing is not a recognized diagnostic tool for food allergy.

- Immunoglobulin G (notably IgG subclass 4) is believed to be a marker of exposure to food and possibly of tolerance. tions among allergy, intolerance and sensitivity to food may not always be clear to patients.

Food allergy is an adverse health effect arising from a specific immune response that occurs reproducibly on exposure to a given food. ${ }^{1}$ Nonimmunologic adverse reactions to food are termed food intolerance and include conditions such as lactase deficiency, dietary protein-induced enterocolitis syndromes and eosinophilic gastrointestinal disease. ${ }^{1}$ Food sensitivity is a nonspecific term that can include any symptom perceived to be related to food and thus may be subject to a wide range of usage and interpretation.

\section{What is tested in blood assays for food sensitivity?}

In testing for food sensitivity, drawn blood is exposed in vitro to a panel of foods and food components. The degree of total immunoglobulin $\mathrm{G}$ (IgG) antibody binding to each food is quantified via enzyme- or fluorescence-linked immunosorbent assays. ${ }^{2}$ Alternatively, IgG subclass 4 (IgG4) binding may be measured in lieu of total IgG.

Results of testing for food sensitivity may be reported to patients as a multipage document that itemizes foods by category. For example, under "dairy" may be listed cheddar cheese, cottage cheese and so on. This is inconsistent with current understanding of food allergy, which has identified substantial allergenic components of cows' milk, for example, casein or $\beta$-lactoglobu$\operatorname{lin}^{3}{ }^{3}$ in contrast to making distinctions between types of cheese made from cows' milk.

Large numbers of foods are tested, many of which are ubiquitous, difficult to avoid or very rarely documented as food allergens (e.g., sugar, yeast). The degree of "sensitivity" or "allergy" is graded by a classification scale pertaining to the photometric measurement of the degree of $\operatorname{IgG}$ binding to each food.

Reports of food sensitivity testing may also include measurement of both food-specific $\mathrm{IgG} 4$ and food-specific immunoglobulin E (IgE) levels to a wide array of foods, which may cause confu- 
sion for patients who purchase the tests. Included with the report may be instructions for patients to strictly or temporarily avoid all foods to which the highest degree of IgG binding is reported. Alternatively, a sample diet may be suggested, with a list of foods to be eaten and avoided on a rotating schedule. Accompanying information may list a range of conditions possibly associated with deposition of allergen-IgG antibody complexes including weight gain, fluid retention, hyperactivity, depression, asthma, hypertension and others.

\section{Don't allergists use a blood test to investigate food allergy?}

Yes, but a different test is ordered: food-specific IgE levels, not IgG or IgG4. Even with food-specific IgE measurements, large "screening" panels are not recommended. Making the diagnosis of a specific food allergy may include the following: a full medical history, physical examination, skin prick testing, carefully selected food-specific IgE levels and oral food challenges to suspected food allergens in some instances. ${ }^{1}$

\section{Is there a relation between food- specific lgG levels and food allergy?}

Research from the early 1980s reported a potential mechanism for IgG4 to trigger basophil histamine release, suggesting a role in the allergic response. Subsequent research clarified that this relation was not limited to IgG4 and in fact involved IgE-receptor cross-linking through a receptor complex found on the basophil membrane. ${ }^{4}$ This supports clinical observations that neither total $\mathrm{IgG}$ nor IgG4 levels correlate with food allergy as shown on double-blind placebocontrolled food challenges. ${ }^{4}$

\section{Are food-specific lgG levels a marker of disease?}

The detection of food-specific IgG shows a physiologic response of the immune system to exposure to food. For IgG4 in particular, this may be the normal human response. ${ }^{5}$ Immunoglobulin $\mathrm{G}$ antibodies directed at specific foods can be found in healthy children as well as adults. ${ }^{6}$ Higher levels of IgG4 to foods in infancy may be associated with tolerance to those foods later in childhood. ${ }^{7}$

The relation between IgG4 and food has been further explored through the recent developments in oral immunotherapy to foods. Efforts to induce permanent tolerance to allergens such as milk or peanuts have been accompanied by increases in patients' IgG4 levels to those foods. ${ }^{8}$ Therefore, IgG4 is believed to be a marker of exposure to food and possibly of tolerance. ${ }^{5}$ Further research is required to clarify any role for specific IgG measurement in the assessment of a patient with potential food allergy.

\section{How should patients be counselled regarding food sensitivity testing?}

Physicians should caution patients about the controversy surrounding testing for food sensitivity. Recent position papers from European and American allergy and immunology societies have emphasized the limitations and potential misuse of IgG4 testing, indicating that these tests are not appropriate for making a diagnosis of food allergy. ${ }^{59}$ Updated guidelines for the diagnosis and management of food allergy list foodspecific IgG4 measurement as an unstandardized and unproven procedure, along with other tests such as hair analysis, cytotoxicity assays or electrodermal ("Vega") testing.

A careful history will often clarify the nature of a patient's or parent's concern and whether it seems to have a consistent or reproducible relation to food. Consultation with a gastroenterologist or allergist-immunologist may be appropriate.

Patients may present for counselling only after testing for food sensitivity has been completed, bringing with them a report and detailed elimination diet plan. The situation may be additionally anxiety-provoking for parents who elected to purchase food sensitivity testing for a dependent child and now must decide whether to follow the instructions on the test report. In this situation, it may be helpful to explain the presence of foodspecific IgG or IgG4 in healthy individuals, and the paucity of evidence for using these tests to diagnose food allergy or guide elimination diets.

\section{Cases revisited}

In Case 1, the patient had been told that dietary exclusions guided by food-specific IgG levels may improve his psoriatic arthritis. The physician describes the relation between IgG levels and exposure to food, and explains that there is no evidence for dietary exclusion in psoriatic arthritis.

Regarding the patient's young child, the doctor explains to the patient that there is no indication for drawing blood from a healthy toddler who has not manifested any signs of adverse reactions to food. Diagnosis of food allergy in a child is associated with increases in caregiver 
stress and anxiety, ${ }^{10}$ and should be based on a thorough clinical history and relevant testing.

In Case 2, the test report recommended many food exclusions, including the child's main source of protein. A recent review of the role of food in atopic dermatitis concluded that a small number of particular foods may provoke exacerbations of atopic dermatitis in a subset (about 35\%) of individuals, and food-specific antibody testing of any kind does not identify these instances. ${ }^{11}$ The physician explains that food challenges should be used to confirm this relation before foods are eliminated from the child's diet and that it is important to keep the nutritional status of the child in mind. ${ }^{11}$ Removing eggs from this child's vegetarian diet would be removing a reliable source of hemebased iron as well as vitamin $B_{12}$. There are insufficient studies on long-term growth to recommend strict vegan diets for children. ${ }^{12}$

\section{References}

1. Boyce JA, Assa'ad A, Burks AW, et al. Guidelines for the diagnosis and management of food allergy in the United States: summary of the NIAID-sponsored expert panel report. J Allergy Clin Immunol 2010;126:1105-18.

2. Terr A. Unconventional theories and unproven methods in allergy. In: Adkinson F Jr, Bochner B, Busse W, et al, editors. Middleton's allergy: principles and practice. 7th ed. Philadelphia (PA): Mosby Elsevier. p. 1691-1706.

3. Natale M, Bisson C, Monti G, et al. Cow's milk allergens identification by two-dimensional immunoblotting and mass spectrometry. Mol Nutr Food Res 2004;48:363-9.

4. Hamilton RG. Clinical laboratory assessment of immediate-type hypersensitivity. J Allergy Clin Immunol 2010;125(Supp 2): S284-96.

5. Bock SA. AAAAI support of the EAACI Position Paper on IgG4. J Allergy Clin Immunol 2010;125:1410.

6. Husby S. Normal immune responses to ingested foods. J Pediatr Gastroenterol Nutr 2000;30(Suppl):S13-9.

7. Tomicic S, Norrman G, Fälth-Magnusson K, et al. High levels of IgG4 antibodies to foods during infancy are associated with tolerance to corresponding foods later in life. Pediatr Allergy Immunol 2009;20:35-41.

8. Jones SM, Pons L, Roberts JL, et al. Clinical efficacy and immune regulation with peanut oral immunotherapy. J Allergy Clin Immunol 2009;124:292-300.

9. Stapel SO, Asero R, Ballmer-Weber BK, et al. Testing for IgG4 against foods is not recommended as a diagnostic tool: EAACI Task Force Report. Allergy 2008;63:793-6.

10. King RM, Knibb RC, Hourihane JO. Impact of peanut allergy on quality of life, stress and anxiety in the family. Allergy 2009; 64:461-8

11. Greenhawt M. The role of food allergy in atopic dermatitis. Allergy Asthma Proc 2010;31:392-7.

12. Amit M. Vegetarian diets in children and adolescents. Paediatr Child Health 2010;15:303-14.

Affiliation: From the Department of Pediatrics, Humber River Regional Hospital, Toronto, Ont. 